\title{
Coherent fans in the space of flows in framed graphs
}

\author{
Vladimir I. Danilov ${ }^{1} \quad$ Alexander V. Karzanov $^{2} \quad$ Gleb A. Koshevoy ${ }^{1}$ \\ ${ }^{1}$ Central Institute of Economics and Mathematics of the RAS, Moscow, Russia \\ ${ }^{2}$ Institute for System Analysis of the RAS, Moscow, Russia
}

\begin{abstract}
Let $G=(V, E)$ be a finite acyclic directed graph. Being motivated by a study of certain aspects of cluster algebras, we are interested in a class of triangulations of the cone of non-negative flows in $G, \mathcal{F}_{+}(G)$. To construct a triangulation, we fix a framing at each inner vertex $v$ of $G$, which consists of two linear orders: one on the set of incoming edges, and the other on the set of outgoing edges of $v$. A digraph $G$ endowed with a framing at each inner vertex is called framed. Given a framing on $G$, we define a reflexive and symmetric binary relation on the set of extreme rays of $\mathcal{F}_{+}(G)$. We prove that that the complex of cliques formed by this binary relation is a pure simplicial complex, and that the cones spanned by cliques constitute a unimodular simplicial regular fan $\Sigma(G)$ covering the entire $\mathcal{F}_{+}(G)$.

Résumé. Soit $G=(V, E)$ un graphe orienté, fini et acyclique. Nous nous intéressons, en lien avec l'étude de certains aspects des algèbres amassées, à une classe de triangulations du cône des flots positifs de $G, \mathcal{F}_{+}(G)$. Pour construire une triangulation, nous ajoutons une structure en chaque sommet interne $v$ de $G$, constituée de deux ordres totaux : l'un sur l'ensemble des arcs entrants, l'autre sur l'ensemble des arcs sortants de $v$. On dit alors que $G$ est structuré. On définit ensuite une relation binaire réflexive et symétrique sur l'ensemble des rayons extrêmes de $\mathcal{F}_{+}(G)$. Nous démontrons que le complexe des cliques formé par cette relation binaire est un complexe simplicial pur, et que le cône engendré par les cliques forme un éventail régulier simplicial unimodulaire $\Sigma(G)$ qui couvre complètement $\mathcal{F}_{+}(G)$.
\end{abstract}

Keywords: Simplicial fan, regular fan, Plücker cluster algebra, coherence binary relation, weakly-separated sets

\section{Introduction}

Let $G=(V, E)$ be a finite acyclic directed graph. We associate to $G$ a vector space $\mathcal{F}(G)$ of flows in $G$ and a cone $\mathcal{F}_{+}(G)$ of nonnegative flows. The extreme rays of the cone $\mathcal{F}_{+}(G)$ correspond to the maximal paths in $G$, called routes, or, equivalently, to the integer flows of value one. Being motivated by a study of certain aspects of cluster algebras, we are interested in a class of triangulations of the cone $\mathcal{F}_{+}(G)$.

To construct a triangulation, we fix a framing at each inner vertex $v$ of $G$, which consists of two linear orders: one on the set of incoming edges, and the other on the set of outgoing edges of $v$. A digraph $G$ endowed with a framing at each inner vertex is called framed. We define a reflexive and symmetric binary relation on the routes of a framed $G$ (or on the set of extreme rays of $\mathcal{F}_{+}(G)$ ). Theorem 1 asserts that the complex of cliques formed by this binary relation is a pure simplicial complex, and that the cones spanned by cliques constitute a unimodular simplicial fan $\Sigma(G)$ covering the entire $\mathcal{F}_{+}(G)$. Theorem 2 asserts that this fan is regular, which means the existence of a convex function on $\mathcal{F}_{+}(G)$ whose affine areas are exactly the maximal cones in the fan.

A route of a framed digraph $G$ is called exceptional if it belongs to each maximal clique. We define the reduced cone $\mathcal{F}_{\text {red }}$ and the reduced fan $\Sigma_{\text {red }}$ to be the quotients of $\mathcal{F}_{+}(G)$ and $\Sigma(G)$, respectively, by the subspace generated by exceptional routes. The fan $\Sigma_{r e d}$ is simplicial and regular as well.

We specify the above results for the class of planar grids forming skew staircase diagrams. For the so-called canonical framing of a skew staircase diagram, the fan $\Sigma_{r e d}$ is complete in the space $\mathcal{F}_{\text {red }}$. This affirmatively answers Conjecture 8.10 in [5].

Next, considering the canonical framing, we study flips between neighboring cones of the fan. Theorem 3 asserts that, for any maximal clique $\mathcal{K}$ and any non-exceptional $A \in \mathcal{K}$, there exists a pair of routes $A^{\prime}, B \in$ $\mathcal{K} \backslash\{A\}$ such that $B^{\prime}=A^{\prime}+B-A$ is a route and $(\mathcal{K} \backslash\{A\}) \cup\left\{B^{\prime}\right\}$ is again a maximal clique.

In the case of staircase diagrams, the routes are identified, in a natural way, with the subsets of the corresponding ground set $[n]=\{1,2, \ldots, n\}$. Using results from [1],we show that any weakly separated set-system

\footnotetext{
${ }^{0}$ Emails: danilovecemi.rssi.ru, sasha@cs.isa.ru,koshevoy@cemi.rssi.ru 
on $[n]$ forms a clique. The cliques of this sort are linked by flips which are associated to certain Plücker relations. Relying on the existence of the Plücker cluster structure on weakly-separated set systems [2],we get a relationship between the fan $\Sigma$ and the Plücker cluster fan. It is shown that for a staircase diagram $\Gamma(n)$ with $n \leq 5$, the cluster fan is a refinement of $\Sigma(\Gamma(n))$, and we conjecture that this is true for any $n$.

\section{Paths, routes and flows}

Let $G=(V, E)$ be a finite acyclic directed graph with vertex set $V$ and edge set $E$. For a vertex $v \in V$, let $i n(v)$ denote the set of incoming edges, and $\operatorname{out}(v)$ the set of outgoing edges. A vertex $v$ is called a source if $\operatorname{in}(v)=\emptyset$, and a sink if $\operatorname{out}(v)=\emptyset$; the other vertices are called inner.

A path in $G$ is a sequence of edges $e_{1}, \ldots, e_{k}$ such that $h\left(e_{i}\right)=t\left(e_{i+1}\right)$ for $i=1, \ldots, k-1$, where $t(e)$ and $h(e)$ denote the beginning and ending vertices (or the tail and the head) of an edge $e$, respectively. Since $G$ is acyclic, any path is simple (i.e. all vertices in it are different). A maximal path is called a route; it begins at a source and ends at a sink. The set of routes is denoted by $\mathcal{P}(G)$, or simply by $\mathcal{P}$. The set of routes passing a vertex $v$ is denoted by $\mathcal{P}(v)$. For $P \in \mathcal{P}(v)$, we write $P v$ for the initial part of $P$, from the beginning to $v$, and write $v P$ for the final part of $P$, from $v$ to the end.

A flow in $G$ is a function $F: E \rightarrow \mathbb{R}$ satisfying the conservation (or Kirchhoff) law at each inner vertex $v$ :

$$
\sum_{e \in i n(v)} F(e)=\sum_{e \in o u t(v)} F(e) .
$$

The set $\mathcal{F}=\mathcal{F}(G)$ of flows in $G$ is a vector subspace of $\mathbb{R}^{E}$. Let $\mathcal{F}_{+}$denote the cone of nonnegative flows, $\mathcal{F}_{\mathbb{Z}}$ the Abelian group of integer flows, and $\mathcal{F}_{1}$ the convex polytope of nonnegative flows $F$ of value one (i.e. satisfying $\sum(F(e): t(e)$ is a source $\left.)=1\right)$. The characteristic function of a route is a vertex of $\mathcal{F}_{1}$, and vice versa. Thus, the set $\mathcal{P}$ can be identified with the set of extreme rays of the cone $\mathcal{F}_{+}$.

The following elementary facts are well-known.

Proposition 1. The dimension of $\mathcal{F}(G)$ is equal to $|E|$ minus the number of inner vertices. The cone $\mathcal{F}_{+}$ spans the vector space $\mathcal{F}$.

Since the cone $\mathcal{F}_{+}(G)$ is defined by the inequalities $F(e) \geq 0$ for $e \in E$, the facets (in $\mathcal{F}(G)$ ) of this cone are of the form $F(e)=0$ for some edges $e$. Let us say that an edge $e$ is idle if at least one takes place: $t(e)$ is inner and $\operatorname{out}(t(e))=\{e\}$, or $h(e)$ is inner and $i n(h(e))=\{e\}$. For an idle edge $e$, the inequality $F(e) \geq 0$ follows from $F(e)=\sum_{e^{\prime} \in \operatorname{in}(t(e))} F\left(e^{\prime}\right)$ in the former case, or from $F(e)=\sum_{e^{\prime} \in \text { out }(h(e))} F\left(e^{\prime}\right)$ in the latter case. Contracting an idle edge, we get a graph which is different from $G$ but has the same flow cone $\mathcal{F}_{+}$in essence. The following fact is easy.

Proposition 2. The set of facet of the cone $\mathcal{F}_{+}(G)$ can be identified with the set of non-idle edges of $G$.

\section{Coherent routes}

A framing on a digraph $G$ is a setting, for each inner vertex $v \in V$, a linear order $\prec_{i n(v)}$ on the set $i n(v)$ and a linear order $\prec_{o u t(v)}$ on the set out $(v)$. A digraph endowed with a framing is called a framed graph, or a net.

For a framed graph $G$ and an inner vertex $v$, we denote by $\operatorname{In}(v)$ the set of maximal paths, or semi-routes, in $G$ entering $v$, and denote by $\operatorname{Out}(v)$ the set of semi-routes leaving $v$. We define a linear order $\prec_{I n(v)}$ on the set $\operatorname{In}(v)$ and a linear order $\prec_{O u t(v)}$ on the set $\operatorname{Out}(v)$ as follows. Suppose that paths $P, Q \in O u t(v)$ coincide before a vertex $w$ (including $w$ ) and differ after $w$ (possibly $w=v$ ). Let $e_{P}$ be the edge of $P$ such that $t\left(e_{P}\right)=w$, and let $e_{Q}$ be a similar edge for $Q$. Then we set $P \prec_{O u t(v)} Q$ if $e_{P} \prec_{\text {out }(w)} e_{Q}$. The linear order $\prec_{\operatorname{In}(v)}$ on $\operatorname{In}(v)$ is defined in a similar way.

Let $P$ and $Q$ be two routes passing an inner vertex $v$. We say that $P, Q$ are in conflict at $v$ if the initial parts $P v, Q v$ are ordered differently than the final parts $v P, v Q$; say, $P v \prec_{I n(v)} Q v$ but $v Q \prec_{O u t(v)} v P$. Otherwise we say that $P$ and $Q$ are coherent at $v$.

Definition. Routes $P$ and $Q$ are called coherent if they are coherent at each common inner vertex.

The following lemma will be of use.

Lemma 1. Let routes $P$ and $Q$ be coherent at some common vertex $v$, and let $D$ be the route obtained by concatenating $P v$ and $v Q$. Suppose that a route $C$ is coherent to $P$ and to $Q$, but not to $D$. Then $C$ and $D$ are conflicting at $v$. 
Proof. If $P v=Q v$ then $D=Q$, and if $v P=v Q$ then $D=P$; in both cases $C$ as above does not exist. Thus, $P v \neq Q v$ and $v P \neq v Q$.

Consider the case $P v \prec_{\operatorname{In}(v)} Q v$. Since $P$ and $Q$ are coherent at $v$, we have $v P \prec_{O u t(v)} v Q$. Since $C$ and $D$ are not coherent, there is a node $w$ at which $C$ and $D$ are conflicting. W.l.o.g., we may assume that $w$ precedes $v$ on the route $D$ (and hence $w$ precedes $v$ on $P$ ). Then $C$ and $D$ are conflicting at $v$ as well. If $C$ and $D$ would become different before $v$, then $C$ and $P$ should be not coherent, contrary to the conditions. The other cases are checked similarly.

Corollary. Under the hypotheses of Lemma 1, assume, in addition, that Pv $\prec_{I n(v)} Q v$. Then Pv $\prec_{\operatorname{In}(v)}$ $C v \preceq_{\operatorname{In}(v)} Q v$ and $v P \preceq_{O u t(v)} v C \prec_{\text {Out }(v)} v Q$.

Proof. The relation $P v \prec_{I n(v)} C v$ follows from the hypotheses of the lemma and the fact that $C$ and $D$ are conflicting at $v$. Since $P$ and $C$ are coherent, we get $v P \preceq O u t(v) v C$. Similarly, $C v \preceq \operatorname{In}(v) Q v$.

The coherence is a reflexive and symmetric binary relation on the set of routes $\mathcal{P}$. We will refer to a set of mutually coherent routes as a clique. Of most interest for us are maximal cliques.

Proposition 3. Let $\mathcal{K}$ be a maximal clique, and let $P, Q \in \mathcal{K}$ be such that:

a) $P$ and $Q$ have a common vertex $v$;

b) $P v$ and $Q v$ are neighbors in the chain $\mathcal{K}(\operatorname{In}(v))=\{P v, P \in \mathcal{K}\}$, and $v P$ and $v Q$ are neighbors in the chain $\mathcal{K}(O u t(v))=\{v P, P \in \mathcal{K}\}$.

Let $D$ denote the route $P v Q$ (obtained by concatenating $P v$ and $v Q$ ), and $F$ the route $Q v P$. Then:

1) $P+Q=D+F$;

2) $D$ and $F$ cannot simultaneously belong to $\mathcal{K}$;

3) if $D \notin \mathcal{K}$, then $F \in \mathcal{K}$, and $\mathcal{K}^{\prime}=(\mathcal{K}-F) \cup D$ is a maximal clique.

Proof. Assertion 1) is obvious. Assertion 2) follows from the fact that $D$ and $F$ are in conflict at $v$. To prove 3), assume that $P v \prec_{\operatorname{In}(v)} Q v$. Since $\mathcal{K}$ is maximal and $D \notin \mathcal{K}$, there is $C \in \mathcal{K}$ such that $D$ and $C$ are in conflict. By the above Corollary, we have $P v \prec_{\operatorname{In}(v)} C v \preceq_{I n(v)} Q v$, and $v P \preceq_{O u t(v)} v C \prec_{O u t(v)} v Q$. Since $P v$ and $Q v$ are neighbors, we have $C v=Q v$. Similarly, $v P=v C$. Hence $C=F$. Similar arguments show that $D$ is coherent to any route in $\mathcal{K}-G$. Thus, $\mathcal{K}^{\prime}=(\mathcal{K}-F) \cup D$ is a clique. Obviously, $\mathcal{K}^{\prime}$ is maximal.

\section{Decomposition Theorem}

Let $G$ be a net (a framed graph). For each clique $\mathcal{C}$, we consider the cone $\operatorname{Con}(\mathcal{C})=\sum_{P \in \mathcal{C}} \mathbb{R}_{+} P$. Clearly $\operatorname{Con}(\mathcal{C}) \subseteq \mathcal{F}_{+}$. When $\mathcal{C}$ runs over the set of cliques, we get a collection of cones. The next theorem asserts that this collection forms a fan $\Sigma$ (the coherent fan) covering the cone $\mathcal{F}_{+}$.

Theorem 1. Let $F \in \mathcal{F}_{+}$be a nonnegative flow in a net $G$. Then there exists a clique $\mathcal{C}$ and a decomposition $F=\sum_{P \in \mathcal{C}} \alpha_{P} P$ with all $\alpha_{P} \geq 0$. Moreover, such a decomposition is unique, and if $F$ is integer-valued, then all numbers $\alpha_{P}$ are integers.

Proof. A) If $G$ is such that all routes are coherent, we are done.

B) Suppose that $G$ contains non-coherent routes, and that no idle edge in $G$ enters a sink. (For deleting such an edge affects neither the cone $\mathcal{F}_{+}$, nor the set of routes, nor any coherent relation on routes.) Since $G$ is acyclic, there exists an inner vertex $v$ such that all edges leaving $v$ enter sinks.

Removing these sinks, we obtain the graph $G^{\prime}=\left(V \backslash \cup_{e \in \text { out }(v)} h(e), E \backslash\right.$ out $\left.(v)\right)$ and take the induced framing on it. The vertex $v$ becomes a sink in $G^{\prime}$.

This gives the corresponding map on routes:

$$
\text { res }: \mathcal{P}=\mathcal{P}(G) \rightarrow \mathcal{P}\left(G^{\prime}\right)=\mathcal{P}^{\prime}
$$

This map is surjective, but need not be injective. One can see that if $\mathcal{C}$ is a clique in $\mathcal{P}$, then its image $\mathcal{C}^{\prime}=$ $\operatorname{res}(\mathcal{C})$ is a clique in $\mathcal{P}^{\prime}$. Moreover, we have a correspondence between the set $\mathcal{C}^{\prime}(v)=\mathcal{C}(\operatorname{In}(v))$ of paths in $G^{\prime}$ ending at $v$ and the set $\operatorname{out}(v)$ of edges leaving $v$ (in $G$ ).

More precisely, consider the set of paths $\mathcal{C}(v)$ in $\mathcal{C}$ passing $v$. Then sending $P v \in \mathcal{C}^{\prime}(v)$ to $v P \in$ out $(v)$ gives the correspondence $\mathcal{C}(v): \mathcal{C}^{\prime}(v) \Longrightarrow$ out $(v)$. Due to the net structure, both sets are ordered. We need the following.

Proposition 4. The correspondence $\mathcal{C}(v): \mathcal{C}^{\prime}(v) \Longrightarrow$ out $(v)$ involves the whole set $\mathcal{C}^{\prime}(v)$ and is monotone with respect to the orders induced by the net $G$. 
Here the proof follows from the facts that the map $\mathcal{C}(v) \rightarrow \mathcal{C}^{\prime}(v)$ is surjective and monotone, and that the map $\mathcal{C}(v) \rightarrow \operatorname{out}(v)$ is monotone. See the picture.

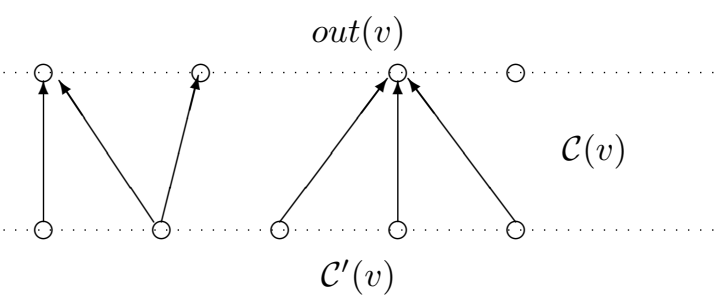

Conversely, any correspondence $R: \mathcal{C}^{\prime}(v) \Longrightarrow$ out $(v)$ which is monotone and defined on the whole set $\mathcal{C}^{\prime}(v)$ determines a natural 'prolongation' of a clique $\mathcal{C}^{\prime}$ in $G^{\prime}$ to some clique $\mathcal{C}$ in $G$. Namely, the subset of routes in $\mathcal{C}^{\prime}$ that avoid $v$ are included in $\mathcal{C}$, and each route $Q^{\prime} \in \mathcal{C}^{\prime}(v)$ (in $G^{\prime}$ ) generates the set $\mathcal{R}\left(Q^{\prime}\right)$ of routes $Q$ (in $G$ ) such that $Q v=Q^{\prime}$ and $v Q \in R\left(Q^{\prime}\right)$. The collection $\mathcal{C}$ is a clique. Indeed, the routes of $\mathcal{C}$ are coherent at any vertex $w \neq v$ as being coherent in $\mathcal{C}^{\prime}$, and they are coherent at $v$ due to the monotonicity of $R$.

Note that for such a prolongation, some routes in $\mathcal{C}^{\prime}(v)$ can be split. This depends on the cardinality of its images in the correspondence $R$. In all cases the size of the clique $\mathcal{C}$ exceeds the size of $\mathcal{C}^{\prime}$ by at most $|\operatorname{out}(v)|-1$. Note that there exists a correspondence for which the size grows by exactly $|\operatorname{out}(v)|-1$. As a consequence, the maximum clique size in $\mathcal{P}$ is equal to $\operatorname{dim}(\mathcal{F})$.

C) Finally, we describe the decomposition of a flow with respect to cliques in $\mathcal{P}(G)$. Let $F$ be a flow in $G$, and let $F^{\prime}=\operatorname{res}(F)$ be its restriction. By induction there is a clique $\mathcal{C}^{\prime}$ in $\operatorname{res}(\mathcal{P})$ and a decomposition of $F^{\prime}$ into a nonnegative linear combination of routes from $\mathcal{C}^{\prime}$.

We construct an appropriate correspondence from $\mathcal{C}^{\prime}(v)$ to out $(v)$ and check the uniqueness of this correspondence. By the conservation low, the value of $F^{\prime}$ entering the sink $v$ (in $G^{\prime}$ ) is equal to $\sum_{e \in o u t(v)} F(e)$. Now the construction is seen from the following picture.

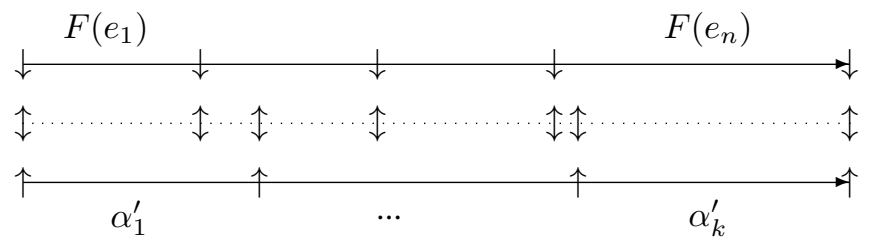

Here $e_{1}<e_{2}<\ldots<e_{n}$ is the ordering of the set out $(v)$ in the net $G, P_{1}^{\prime}<\ldots<P_{k}^{\prime}$ is the ordering of routes in $\mathcal{C}^{\prime}(v)$, and $\alpha_{i}^{\prime}$ denotes the weight of the route $P_{i}^{\prime} \in \mathcal{C}^{\prime}(v)$ in the decomposition of $F^{\prime}$ in the clique $\mathcal{C}^{\prime}$, $i=1, \ldots, k$.

This gives the required monotone correspondence, yielding uniquely the prolongation of $\mathcal{C}^{\prime}$ to $\mathcal{C}$ and the decomposition of $F$ over $\mathcal{C}$. The integrality result easily follows.

In particular, from Theorem 1 it follows that for any maximal clique $\mathcal{K}$, the routes in $\mathcal{K}$ form a basis of the Abelian group $\mathcal{F}_{\mathbb{Z}}$ of integer flows. For particular staircase diagram graphs, such a property was proved in [5].

\section{Regularity of the fan of cliques}

For a net $G$, consider the fan $\Sigma$ of cliques in $\mathcal{P}(G)$. The regularity of $\Sigma$ means that there exists a homogeneous concave function $w: \mathcal{F}_{+} \rightarrow \mathbb{R}$ whose linearity areas are exactly the clique cones in $\Sigma$. Extending a result in [5],we show the following

Theorem 2. For an arbitrary net $G$, the fan $\Sigma$ of cliques in $\mathcal{P}(G)$ is regular.

Proof. We explicitly construct a required concave function. Since $\Sigma$ is formed by simplicial cones, it suffices to define values $w(P)$ on the routes $P \in \mathcal{P}$. Then for each flow $F=\sum_{P \in \mathcal{C}} \alpha_{P} P$, where $\mathcal{C}$ is a clique and all $\alpha_{P} \geq 0$, we define $\widetilde{w}(F):=\sum \alpha_{P} w(P)$.

We have to ensure that the resulting $\widetilde{w}$ is concave and, moreover, it is strictly concave with respect to the fan $\Sigma$. The latter can be described as follows. Let $\operatorname{Con}(\mathcal{C})$ be a full dimensional cone in $\Sigma$, and let $l_{\mathcal{C}}$ be the unique linear function on $\mathcal{F}$ taking the values $w(P)$ for all $P \in \mathcal{C}$. Then any flow $F \notin C o n(\mathcal{C})$ should satisfy the strict inequality $\widetilde{w}(F)<l_{\mathcal{C}}(F)$. 
Lemma 2. Let $w: \mathcal{P} \rightarrow \mathbb{R}$ be a function satisfying the following condition:

(*) for any two non-coherent routes $P$ and $Q$, there exist routes $P^{\prime}$ and $Q^{\prime}$ such that $P+Q=P^{\prime}+Q^{\prime}$ and $w(P)+w(Q)<w\left(P^{\prime}\right)+w\left(Q^{\prime}\right)$.

Then the piecewise linear extension $\widetilde{w}$ is strictly concave w.r.t. the fan $\Sigma$.

Proof of Lemma 2. Consider a concave interpolation $\widehat{w}$ of $w$, i.e. for each flow $F$, the value $\widehat{w}(F)$ is the supremum of values $\sum \alpha_{P} w(P)$ over the decompositions $\sum \alpha_{P} P=F$ with nonnegative coefficients $\alpha=\left(\alpha_{P}\right), P \in \mathcal{P}$. Such $\alpha$ 's constitute a compact set, and hence the supremum is attained at some of these decompositions. Let $\alpha=\left(\alpha_{P}\right)$ be the tuple of coefficients in it. We claim that

The collection $\mathcal{C}$ of those $P \in \mathcal{P}$ where $\alpha_{P}>0$ is a clique.

Indeed, if $\mathcal{C}$ contains a non-coherent pair $P, Q$, then we can achieve a bigger value of $\widehat{w}(F)$ by involving the pair $P^{\prime}, Q^{\prime}$ as in condition $(*)$.

It follows that $\widetilde{w}$ and $\widehat{w}$ coincide, implying the concavity of $w$. To show the strict concavity, consider a maximal clique $\mathcal{K}$ and the corresponding cone $\operatorname{Con}(\mathcal{K})$. W.1.o.g. we may assume that the linear function $l_{\mathcal{K}}$ is zero on $\operatorname{Con}(\mathcal{K})$. Then for any $P \notin \mathcal{K}$, the concavity of $\widehat{w}$ implies $w(P) \leq 0$. We have to show that $w(P)<0$.

Take a small $\varepsilon>0$ so that the flow $F=\sum_{K \in \mathcal{K}} K+\varepsilon P$ belong to the cone $C o n(\mathcal{K})$, and hence $\widehat{w}(F)=0$. Since $\mathcal{K}$ is a maximal clique and $P \notin \mathcal{K}$, there exists a route $Q \in \mathcal{K}$ such that $Q, P$ are not coherent. Let $P^{\prime}, Q^{\prime}$ be as in $(*)$ and consider the decomposition

$$
F=\sum_{K \in \mathcal{K}, K \neq Q} K+(1-\varepsilon) Q+\varepsilon\left(P^{\prime}+Q^{\prime}\right) .
$$

Since $\widehat{w}(F)=0$, we have

$$
\sum_{K \in \mathcal{K}, K \neq Q} w(K)+(1-\varepsilon) w(Q)+\varepsilon\left(w\left(P^{\prime}\right)+w\left(Q^{\prime}\right)\right) \leq 0 .
$$

Since $w(K)=0$ for any $K \in \mathcal{K}$, we obtain $w\left(P^{\prime}\right)+w\left(Q^{\prime}\right) \leq 0$. Now $(*)$ and $w(Q)=0$ imply $w(P)<0$, proving the lemma.

To construct the desired $w$ on $\mathcal{P}$, we consider a route $P$ and define numbers $\delta_{k}(e)=\delta_{k}(e, P)$ for each $k \geq 1$ and each edge $e$ of $P$ as follows. Let $n(e)$ be the number of $e$ in the order $\prec_{i n(h(e))}$. Let $e^{\prime}$ be the $k$-th successor of $e$ in $P$ (if $e^{\prime}$ does not exist, we set $\delta_{k}(e):=0$ ), and let $n\left(e^{\prime}\right)$ be the number of $e^{\prime}$ in the order $\prec_{\text {out }}\left(t\left(e^{\prime}\right)\right)$. We define

$$
\delta_{k}(e):=\left(n(e)+n\left(e^{\prime}\right)\right)^{2} \quad \text { and } \quad w_{k}(P):=\sum_{e \in P} \delta_{k}(e) .
$$

Finally, taking a small positive real $\varepsilon$, define

$$
w(P):=w_{1}(P) \varepsilon+w_{2}(P) \varepsilon^{2}+\ldots
$$

Lemma 3. The function $w: \mathcal{P} \rightarrow \mathbb{R}$ defined in (1) satisfy condition $(*)$.

(Then Lemmas 2 and 3 imply Theorem 2.)

Proof of Lemma 3. Let $P$ and $Q$ be conflicting at some vertex $v$, say, $P v \prec_{I n(v)} Q v$ and $v Q \prec$ Out (v) $v P$. Denote by $K$ the maximal common subpath of $P$ and $Q$ containing $v$. Let $K=\left(e_{1}, \ldots, e_{k-1}\right)$. Denote by $e_{P}$ the edge of $P$ such that $h\left(e_{P}\right)=t\left(e_{1}\right)$, and denote by $f_{P}$ the edge of $P$ such that $h\left(e_{k-1}\right)=t\left(f_{P}\right)$. Denote by $e_{Q}$ and $f_{Q}$ the corresponding edges for $Q$. See the picture.

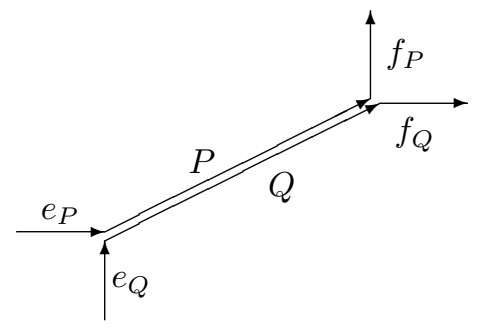


Let $P^{\prime}:=P v Q$ (the concatenation of $P v$ and $v Q$ ), and $Q^{\prime}:=Q v P$. Obviously, $P+Q=P^{\prime}+Q^{\prime}$. We claim that

$$
w\left(P^{\prime}\right)+w\left(Q^{\prime}\right)>w(P)+w(Q) .
$$

1) If $l<k$ then

$$
w_{l}(P)+w_{l}(Q)=w_{l}\left(P^{\prime}\right)+w_{l}\left(Q^{\prime}\right) .
$$

Indeed, let $e$ and $e^{\prime}$ be edges of $P$ such that the subpath $L=\left(e, \ldots, e^{\prime}\right)$ of $P$ has length $l$. Then $K$ cannot be a proper subpath of $L$. Therefore, $L$ is contained either in $P^{\prime}$ or in $Q^{\prime}$, and 3 is valid.

2) Let $l=k$. Arguing as above, one can see that the difference $w_{k}(P)+w_{k}(Q)-\left(w_{k}\left(P^{\prime}\right)+w_{k}\left(Q^{\prime}\right)\right)$ is equal to

$$
\left(n\left(e_{P}\right)+n\left(f_{P}\right)\right)^{2}+\left(n\left(e_{Q}\right)+n\left(f_{Q}\right)\right)^{2}-\left(\left(n\left(e_{P}\right)+n\left(f_{Q}\right)\right)^{2}+\left(n\left(e_{Q}\right)+n\left(f_{P}\right)\right)^{2}\right) .
$$

Denote $a:=n\left(e_{P}\right), b:=n\left(f_{P}\right), c:=n\left(e_{Q}\right)$, and $d:=n\left(f_{Q}\right)$. Since $P, Q$ are conflicting at $v$, we have $a<c$ and $d<b$. Therefore,

$$
(a+b)^{2}+(c+d)^{2}-\left((a+d)^{2}+(c+b)^{2}\right)=-2(a-c)(d-b)<0 .
$$

This implies

$$
w_{k}(P)+w_{k}(Q)<w_{k}\left(P^{\prime}\right)+w_{k}\left(Q^{\prime}\right) .
$$

3) Let $l>k$. In this case we cannot estimate the difference $w_{l}(P)+w_{l}(Q)-\left(w_{l}\left(P^{\prime}\right)+w_{l}\left(Q^{\prime}\right)\right)$, but we can choose $\varepsilon$ so that the "tail" $\sum_{l>k} \varepsilon^{l}\left(w_{l}(P)+w_{l}(Q)-\left(w_{l}\left(P^{\prime}\right)+w_{l}\left(Q^{\prime}\right)\right)\right)$ provide the negativity of the "head": $\varepsilon\left(w_{k}(P)+w_{k}(Q)-\left(w_{k}\left(P^{\prime}\right)+w_{k}\left(Q^{\prime}\right)\right)\right)=\sum_{l \leq k} \varepsilon^{l}\left(w_{l}(P)+w_{l}(Q)-\left(w_{l}\left(P^{\prime}\right)+w_{l}\left(Q^{\prime}\right)\right)\right)<0$. This gives 22).

Remark. The set of regular triangulations of the polytope $\mathcal{F}_{1}(G)$ is identified with the the set of vertices of the corresponding secondary polytope [3]. Because of above theorems, we have that each framing on $G$ corresponds to a vertex of the secondary polytope. Does the set of such vertices form a proper subset of vertices of the secondary polytope or not?

\section{Reduced picture}

A route $P$ in a net $G$ is said to be exceptional if $P$ is coherent to each route in $G$. Let $\mathcal{E}$ be the set of exceptional routes. Then $\mathcal{E}$ lies in each maximal clique. The reduced picture arises when we apply factorization by the subspace $\mathbb{R} \mathcal{E}$. Let $\mathcal{F}_{\text {red }}:=\mathcal{F} / \mathbb{R} \mathcal{E}$ be the corresponding factor space, and $p: \mathcal{F} \rightarrow \mathcal{F}_{\text {red }}$ the canonical projection. This determines the reduced cone $\mathcal{F}_{+, \text {red }}=p\left(\mathcal{F}_{+}\right)$in the space $\mathcal{F}_{\text {red }}$ and the reduced fan $\Sigma_{\text {red }}$ consisting of simplicial cones $\sigma(\mathcal{C}):=p(\operatorname{Con}(\mathcal{C}))$.

The reduced fan $\Sigma_{\text {red }}$ is regular. This follows from Theorem 2. Indeed, let $w: \mathcal{F}_{+} \rightarrow \mathbb{R}$ be a strictly concave function (w.r.t. $\Sigma$ ). Considering $w$ up to subtracting a linear function $l$ such that $l(P)=w(P)$ for all $P \in \mathcal{E}$, we may assume that $w(P)=0$ for any $P \in \mathcal{E}$. Then $w=p^{*}(\widetilde{w})$ for some function $\widetilde{w}$ on the reduced cone $\mathcal{F}_{+, \text {red }}$, and $\widetilde{w}$ is strictly concave w.r.t. the fan $\Sigma_{r e d}$. So $\Sigma_{r e d}$ is regular.

This construction is of interest when $\mathcal{E}$ is "large".

Definition. We say that the set $\mathcal{E}$ is ample if it is not contained in any facet of $\mathcal{F}_{+}$.

Proposition 5. The following statements are equivalent:

1) $\mathcal{E}$ is ample;

2) the reduced cone $\mathcal{F}_{+, \text {red }}$ is the whole space $\mathcal{F}_{\text {red }}$;

3) each non-idle edge belongs to an exceptional route.

Proof. If $\mathcal{E}$ lies in none of the facets of the cone $\mathcal{F}_{+}$, then 0 lies in none of the facets the reduced cone $\mathcal{F}_{+, \text {red }}$. Hence 0 is an interior point of $\mathcal{F}_{+, \text {red }}$, implying that this cone coincides with whole space $\mathcal{F}_{\text {red }}$. The converse is also true. Thus, 1) and 2) are equivalent.

The equivalence of 1) and 3) follows from Proposition 2.

Note that there are nets with empty $\mathcal{E}$. In the next section we will consider a class of "grid nets" which possess ample exceptional sets. 


\section{Canonical framing on grid nets}

Let $\lambda=\left(\lambda_{1} \geq \ldots \geq \lambda_{n}\right)$ and $\mu=\left(\mu_{1} \geq \ldots \geq \mu_{n}\right)$ be two partitions and $\lambda \geq \mu$ (i.e. $\lambda_{i} \geq \mu_{i}$ for each $i$ ). Denote by $\Gamma=\Gamma(\lambda, \mu)$ the digraph (called a skew staircase diagram, or a grid net) being the union of directed unit squares in $\mathbb{Z}^{2}$ (where the edges are directed to the east and north) corresponding to the skew Young diagram for $\lambda, \mu$ drawn in the French style. The sources are located at the south-west corners and the sinks are located at the north-west corners of $\Gamma$. The picture below illustrates $\Gamma((3,2,1),(1,0,0))$.

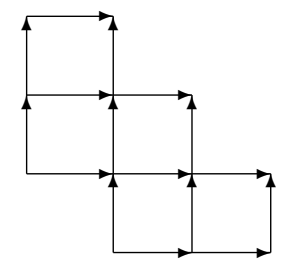

Definition. We say that a framing on a grid net $\Gamma$ is canonical if the vertical edge is bigger than the horizontal one in each corresponding local order.

In the rest of the paper we consider only canonical grid nets.

For the canonical framing on $\Gamma(\lambda, \mu)$, the exceptional routes are of two types: horizontal and vertical. Namely, consider a horizontal edge of $\Gamma$ (which is directed east). Then there exists a unique route which contains this edge and goes horizontally between the SW and NE boundaries of $\Gamma$. Similarly, for each vertical edge (directed north), there is a unique route which contains this edge and goes vertically between the SW and $\mathrm{NE}$ boundaries of $\Gamma$. Example of horizontal- and vertical-type routes are depicted below.

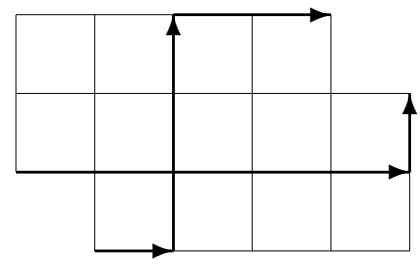

The following lemma is a slight generalization of Lemma 6.8 in [5].

Lemma 4. Any horizontal- or vertical-type route as defined above is exceptional.

Proof. Consider a horizontal-type route $H$; for a vertical-type route, the argument is similar. For any route $P$, there is at most one component of $P \cap H$ containing neither the source nor the sink of $H$. This component has only horizontal edges, and now the fact that $P$ and $H$ are coherent easily follows.

Since the exceptional routes cover all edges, Proposition 5 implies the following

Corollary. For the net $\Gamma(\lambda, \mu)$, the cones in the reduced fan $\Sigma(\Gamma(\lambda, \mu))_{\text {red }}$ cover the reduced vector space $\mathcal{F}_{\text {red. }}$. Hence the reduced fan is complete and regular.

This affirmatively answers Conjecture 8.10 in [5].

Let us return to the fan $\Sigma(\Gamma(\lambda, \mu))$. Consider a maximal clique $\mathcal{K}$ and a route $A \in \mathcal{K}$. This route generates the extreme ray $\mathbb{R}_{+} A$ of the simplicial cone $\operatorname{Con}(\mathcal{K})$. Also $\operatorname{Con}(\mathcal{K}-A)$ is the facet opposite to $A$ in $\operatorname{Con}(\mathcal{K})$. If this facet is not included in a facet of $\mathcal{F}_{+}(\Gamma(\lambda, \mu))$, then it belongs to another full dimensional simplicial cone $\operatorname{Con}\left(\mathcal{K}^{\prime}\right)$. Hence there is a route $B^{\prime}$ such that $\mathcal{K}^{\prime}=(\mathcal{K}-A) \cup B^{\prime}$. The transformation of $\operatorname{Con}(\mathcal{K})$ into $\operatorname{Con}\left(\mathcal{K}^{\prime}\right)$ is called a flip at $A$.

Since any maximal clique contains all exceptional routes, such routes cannot be used in flips. If $A \in \mathcal{K}$ is non-exceptional, then, due to the above Corollary, the cone spanned by $\mathcal{K}-A$ is not contained in a facet of $\mathcal{F}_{+}$, and hence we can make a flip at $A$. This implies that in the reduced fan $\Sigma_{r e d}$, one can make a flip at each element.

Theorem 3. Let $\mathcal{K}$ be a maximal clique in the net $\Gamma(\lambda, \mu)$, and let $A \in \mathcal{K}$ be a non-exceptional route. Then there exist routes $A^{\prime}, B \in \mathcal{K}-\{A\}$ such that $B^{\prime}=A^{\prime}+B-A$ is a route and the collection $\mathcal{K}^{\prime}=(\mathcal{K}-A) \cup B^{\prime}$ is a maximal clique. 
To prove this theorem, we explicitly construct the routes $A^{\prime}$ and $B$.

The construction of $A^{\prime}$. We say that a route $\widetilde{A} \neq A$ is a fellow-traveler of $A$ if $\widetilde{A}$ belongs to $\mathcal{K}$ and has the same source as $A$. Among the fellow-travelers of $A$, we consider the set $\mathcal{A}$ of routes which have the longest common path with $A$ starting at the source of $A$. Let $v$ be the last vertex of this path. (Clearly $v$ is an inner vertex.) Then we take as $A^{\prime}$ the route in $\mathcal{A}$ which is closest to $A$ in the order $\prec$ Out $(v)$.

Suppose that the edge of $A^{\prime}$ leaving $v$ is vertical, while the edge of $A$ leaving $v$ is horizontal.

The construction of $B$. Let $\mathcal{B}$ be the subset of routes $\widetilde{B} \neq A$ in $\mathcal{K}$ satisfying the following conditions:

1. $\widetilde{B}$ contains $v$ and its edge leaving $v$ is horizontal;

2. $\widetilde{B} \prec_{\operatorname{In}(v)} A$.

Note that the set $\mathcal{B}$ is nonempty. Indeed, $\mathcal{B} \cup\{A\}$ contains the horizontal-type exceptional route $E$ passing through the horizontal edge leaving $v$. It suffices to check that $E v \neq A v$. If this is not so, then either $E$ is a longer fellow-traveler of $A$ than the route $A^{\prime}$, or $A$ is an exceptional route; both cases are impossible. Thus, $\mathcal{B}$ is nonempty.

Let $B$ be a minimal element of $\mathcal{B}$ w.r.t. the order $\prec_{\operatorname{In}(v)}$. If there are several such elements, we take as $B$ the one which is maximal w.r.t. the order $\prec_{O u t(v)}$.

We claim that $v B$ coincides with $v A$.

Indeed, since $B$ and $A$ are coherent and $B \prec_{I n(v)} A$, we have $B \preceq_{O u t(v)} A$. We show that the strict comparison here is impossible, as follows.

Suppose that $B \prec_{O u t(v)} A$, and let $D=A v B$. Then $D \neq A$. The route $D$ does not belong to the clique $\mathcal{K}$, otherwise $D$ would be a longer fellow-traveler of $A$ than $A^{\prime}$.

By the Corollary from Lemma 1, there exists a route $C \in \mathcal{K}$ located "between" $A$ and $B$ such that: the first edges of $C$ and $A$ are different, and the last edges of $C$ and $B$ are different. One can see that $C \in \mathcal{B}$. Since $B$ is maximal in $\mathcal{B}$ w.r.t. $\prec_{I n(v)}$, we get $C v=B v$. But then $B \prec_{O u t(v)} C$, contradicting the choice of $B$. This proves the claim.

Now Theorem 3 follows from Proposition 3.

\section{Weakly separated set-systems and Plücker cluster algebra}

Consider a staircase net $\Gamma(n)$ being the canonically framed grid $\Gamma((n-1, \ldots, 1),(0, \ldots, 0))$. Below the net $\Gamma(4)$ is depicted.

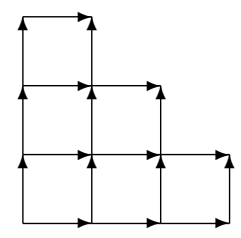

The dimension of the cone $\mathcal{F}_{+}(\Gamma(n))$ is $n(n+1) / 2-1$ and its extreme rays can be identified with $2^{[n]} \backslash\{\emptyset, n\}$. In fact, routes in $\Gamma(n)$ can be identified with words of length $n$ in the alphabet $\{N, E\}$. There is a bijection between the set of such words and the set of subsets of $[n]$ minus $\emptyset$ and $[n]$. Namely, a word is sent into a subset indexing the letter $N$ in the word. For example, the word $N E E N$ is sent to a subset $\{1,4\}$.

Thus any clique of the coherence relation gives a subset in $2^{[n]}$ (or a set-system in $[n]$ ).

Some of such cliques corresponds to seed into the Plücker cluster algebra of the coordinate ring of the flag variety. Namely, Leclerc and Zelevinsky in [4],in connection with the problem of characterizing quasicommuting quantum flag minors, introduced a reflexive and symmetric binary relation on subsets of $[n]$, the so-called weak separation. It is not difficult to show that the cliques of this complex constitute a subset of cliques of the coherence complex for the canonical framing on $\Gamma(n)$. Because of main result in [1], the cliques complex of this binary relation is pure of the dimension $n(n+1) / 2-1$. Thus, we get a pure subcomplex of $\Sigma(\Gamma(n))$ of the same dimension. In [2],we established that maximal weakly-separated set-systems in $[n]$ correspond to seeds into the Plücker cluster algebra. Thus, such 'Plücker seeds' correspond to cones in the coherence fan $\Sigma(\Gamma(n))$.

We illustrate this relation by an example with $n=4$. 


\subsection{Example $n=4$}

Consider $\Gamma(4)$. There are 14 sets-routes $2^{[4]} \backslash\{\emptyset,[4]\}$. Six of them, 1, 12, 123, 234, 34 and 4 constitute the exceptional set $\mathcal{E}(\Gamma(4))$. The non-exceptional sets-routes are 2, 3, 24, 14, 23, 13, 134, and 124 . We implement the non-exceptional routes by vectors in the three-dimensional reduced space $\mathcal{F}(\Gamma(4))_{\text {red }}=\mathcal{F}(\Gamma(4)) / \mathbb{R} \mathcal{E}(\Gamma(4))$.

In this space $\mathcal{F}(\Gamma(4))_{\text {red }}$ the images of routes are related by the following relations (these relations follows from the corresponding relations in $\mathcal{F}(\Gamma(4))$ :

$$
\begin{aligned}
& \overline{3}+\overline{14}=0 \text { (because there holds } 3+14=1+34 \text { in } \mathcal{F}) \\
& \overline{2}+\overline{134}=0(2+134=1+234) \\
& \overline{23}+\overline{124}=0(23+124=12+234) \\
& \overline{2}+\overline{14}=\overline{24}(2+14=1+24) \\
& \overline{23}+\overline{134}=\overline{13}(23+134=13+234) .
\end{aligned}
$$

Let the vectors $\overline{3}, \overline{23}$ and $\overline{2}$ be a basis of the 3 -dim space $\mathcal{F}(\Gamma(4))_{\text {red }}$. Then eight non-exceptional vectors are of the form

$\overline{3}=(1,0,0), \overline{14}=(-1,0,0), \overline{23}=(0,1,0), \overline{124}=(0,-1,0), \overline{2}=(0,0,1), \overline{134}=(0,0,-1), \overline{24}=$ $(-1,0,1), \overline{13}=(0,1,-1)$.

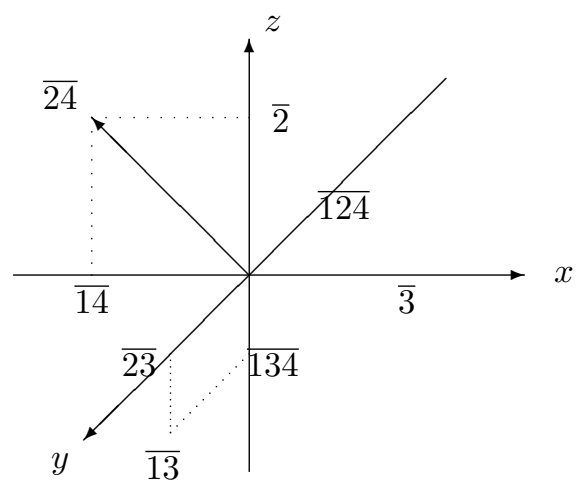

The reduced fan $\Sigma(\Gamma(4))_{\text {red }}$ is the normal fan to the following polytope

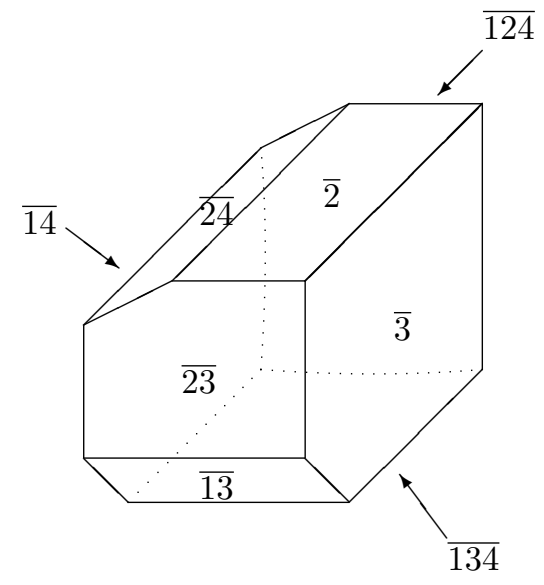

Figure 1

This fan as a simplicial complex is depicted below (the triangle $(14,124,134)$ is also a simplex of the complex). 


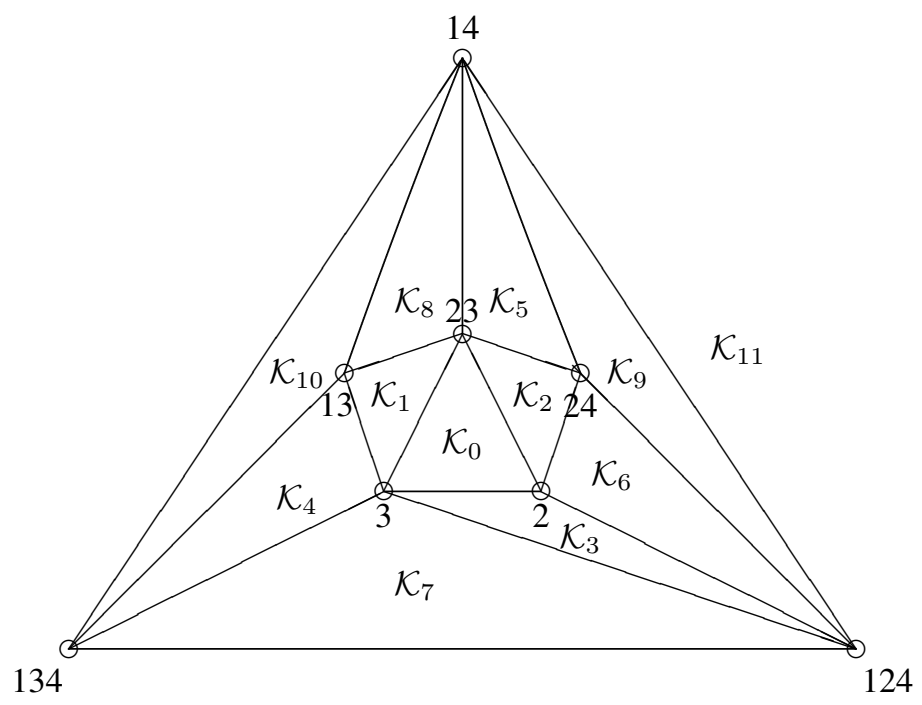

Figure 2

All cliques except two, $\mathcal{K}_{3}$ and $\mathcal{K}_{7}$, correspond to maximal weakly-separated sets. Let us make the baricentric subdivision of the complex (Fig. 2) in the middle of the edge $(3,124)$, which separates the cliques $\mathcal{K}_{3}$ and $\mathcal{K}_{7}$ (this corresponds to cut away the north-east edge of the facet $\overline{3}$ of the polytope (Fig. 1)). Then the resulting fan will be the cluster fan for $S L(4) / N$. This fan is the normal fan to the Postnikov associahedron. The Postnikov associahedron differs from the Chapoton-Fomin-Zelevinsky associahedron, see [6].

\section{References}

[1] V.I. Danilov, A.V. Karzanov, and G.A. Koshevoy, On maximal weakly separated set-systems, J. Algebraic Combin. 32 (4) (2010), 497-531.

[2] V.I. Danilov, A.V. Karzanov, and G.A. Koshevoy, Generalized tilings and Plücker cluster algebras, in Proc. of 7th Int. Conference on Lattice Path Combinatorics and Applications, Siena University, 2010.

[3] I.M. Gelfand, M.M.Kapranov, and A.Zelevinsky, Discriminants, Resultants and Multidimensional Determinants, Birkhäuser, Boston, MA, 1994.

[4] B. Leclerc and A. Zelevinsky, Quasicommuting families of quantum Plücker coordinates, Amer. Math. Soc. Trans., Ser. 2, 181 (1998) 85-108.

[5] T.K. Petersen, P. Pylyavskyy, and D.E. Speyer, A non-crossing standard monomial theory, ArXiv:0806.1776.

[6] C. Ceballos and G.M. Ziegler, Realizing the associahedron: Mysteries and questions, ArXiv:1110.4059. 